\title{
NÉCESSITÉ DE TROIS PARAMÈTRES \\ POUR LAA CLASSIFICATION PRÉCISE \\ DES ÉTOILES DES PREMIERS TYPES SPECTRAUX
}

\author{
D. CHALONGE
}

(exposé d'ensemble de recherches effectuées à l'Institut d'Astrophysique de Paris, notamment par Jacques BERGER)

\section{RÉ S U M É}

Une classification stellaire utilisant trois paramètres quantitatifs a été construite : elle permet de classer des étoiles qui n'avaient pas place dans les anciens systèmes.

Une classification précise exige trois paramètres aussi bien pour la population I que pour la population II. Lorsque les valeurs des trois paramètres caractérisant une étoile sont connues, sa magnitude absolue semble déterminée sans ambiguité : ainsi toute " dispersion cosmique " disparaîtrait. Cette notion serait la conséquence du manque de précision de la classification à deux paramètres qui a été employée jusqu'ici.

\section{N T R O D U C T I O N}

L'introduction d'un troisième paramètre dans la classification stellaire a eu pour objet initial de permettre la classification des étoiles qui ne pouvaient entrer dans les systèmes à deux paramètres, notamment les étoiles à raies métalliques et les étoiles de la population II.

La Figure I rappelle la définition des trois paramètres choisis pour caractériser une étoile : la grandeur $\mathrm{D}$ de la discontinuité de Balmer, une longueur d'onde $\lambda_{1}$ liée à la position de cette discontinuité dans le spectre et le gradient absolu $\Phi_{\mathrm{b}}$ pour le domaine spectral 3800-4800 $\AA$.

Chaque étoile peut ainsi être représentée par un point de l'espace ayant pour coordonnées les valeurs des trois paramètres qui lui correspondent et, dans cet espace $\mathrm{E}$, les points représentatifs doivent se grouper suivant les proprié- respectivement les fonds continus visible et ultraviolet.

tés des étoiles correspondantes. La discontinuité de Balmer est $\mathrm{D}=\log \frac{\text { intensité en } \mathrm{C}}{\text { intensité enD }}, \quad \lambda_{1}$ est la longueur

Une première détermipeunation rapide, et par suite, précise, des paramètres relatifs

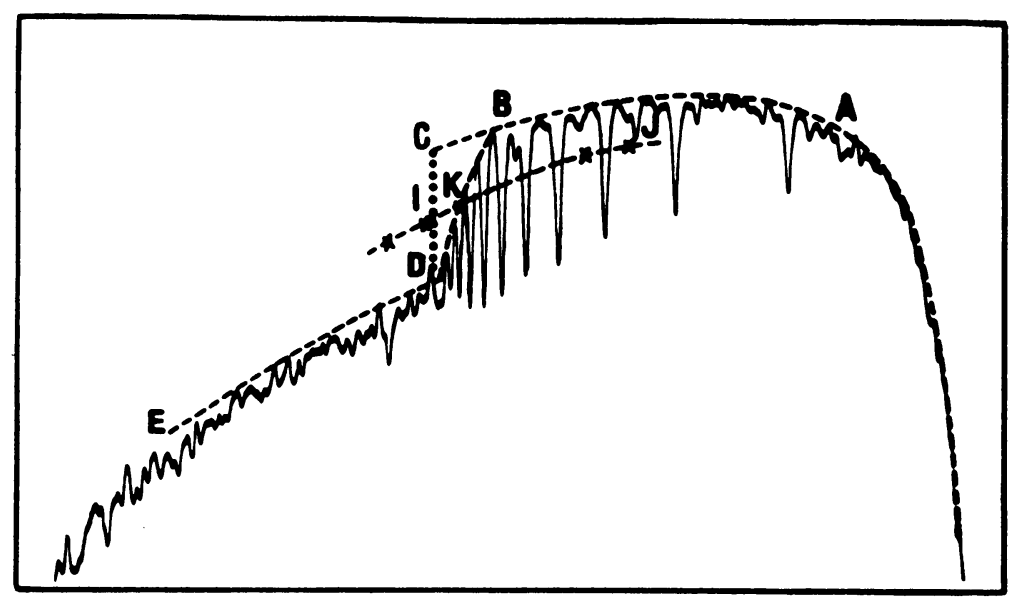

FIGURE I. Définition des trois paramètres.

d'onde du point $K$, équidistant des deux fonds continus, $\Phi_{b}=\frac{C_{2}}{T_{b}}$ $\left(\mathrm{I}-\mathrm{e}^{\left.-\mathrm{C}_{2} / \lambda \mathrm{T}_{\mathrm{b}}\right)-1}\right.$ où $\mathrm{T}_{\mathrm{b}}$ est la température de couleur pour le domaine $\mathrm{ABC}$ et $\lambda$ une longueur d'onde moyenne. 
à un certain nombre d'étoiles permit de constater que celles des types $\mathrm{O}$ à $\mathrm{F}$ (population $\mathrm{I}$ ) qui trouvent place dans les classifications usuelles, étaient représentées dans ce nouveau système par des points voisins d'une surface $\Sigma$ (Fig. 2) (pour plus de détails, voir [I]).

Ce résultat parut normal : les points figuratifs d'étoiles qui entrent dans une classification à deux paramètres (comme la classification MK) devraient en effet se grouper, non dans un volume, mais sur une surface et les écarts constatés entre les points et la surface furent



La figure représente un modèle de la surface qui a été creusé dans un parallélépipède dont les arêtes sont parallèles aux axes de coordonnées $\lambda_{1}-3700$ (et non pas $\lambda_{1}$ comme il est indiqué par erreur sur $\Sigma$ ), D et $\Phi_{\mathrm{b}}$.

Les courbes qui séparent les types spectraux sont les intersections de $\Sigma$ avec une série de plans perpendiculaires à l'axe $\Phi_{b}$. Une autre série de courbes grossièrement orthogonales à celles-ci séparent les diverses classes de luminosité.

L'espace défini par les coordonnées $\lambda_{1}, D, \Phi_{b}$ est désigné sous le nom d'espace E.

d'abord attribués à des erreurs dans la détermination des trois coordonnées. Cependant, une classification plus précise ne tarda pas à nous révéler que les écarts observés étaient réels : les étoiles étaient représentées en fait, non par les points de la surface mais par les points d'un volume enveloppant $\Sigma$. Une classification correcte exigeait donc trois paramètres pour classer la population I (I).

Il est plus facile de démontrer ce résultat pour les naines des classes $\mathrm{F}$ et $\mathrm{A}$ que pour les types moins avancés ( $\mathrm{O}$ et $\mathrm{B})$ : l'absorption interstellaire complique en effet beaucoup la déter-

(I) Quant aux étoiles de la population II, elles s'étaient tout de suite placées nettement en dehors de $\Sigma$. 


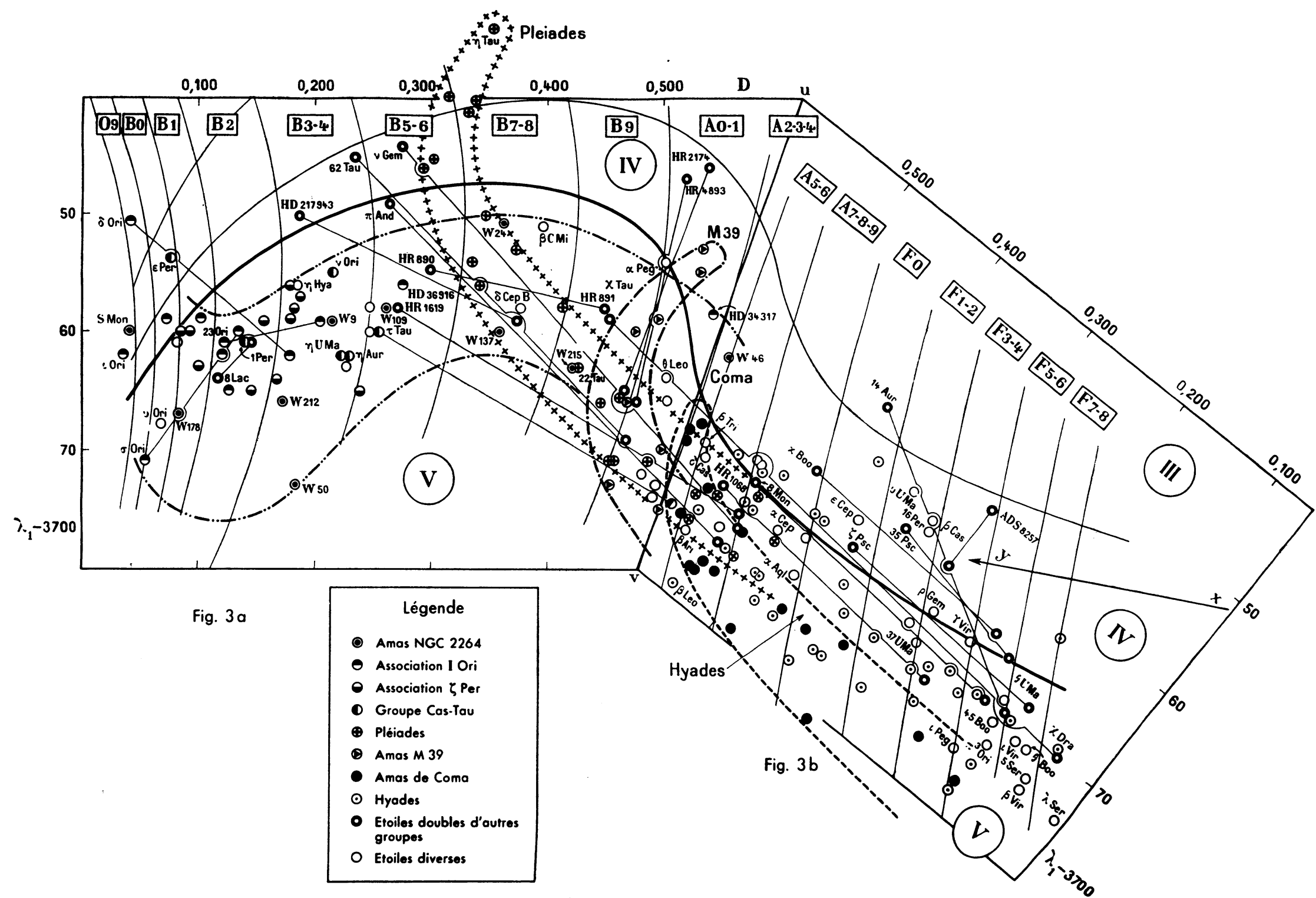

Figure 3 (a). Projection sur le plan PQR (FIG. 2) parallele aux axes $\lambda_{1}$ et $D$ de la partie gauche de la surface $\Sigma$ (types moins avancés que $\mathrm{A}_{3-4}$, $\Phi_{\mathrm{b}}<\mathrm{I}, 30$ ).

FIGURE 3 (b). Projection sur le plan STU (FIG. 2) parallele aux axes $\lambda_{1}$ et $D$ de la partie droite de la surface (types plus avancés que A2, $\Phi_{\mathrm{b}}>$ I.30).

Les deux figures sont juxtaposées le long d'une droite qui est à peu près la projection de la ligne de séparation entre les types A2 et A3-4 (pour les classes de luminosité IV et V).

La figure $3(\mathrm{a} b)$ peut être comparée à un diagramme HR : les types spectraux sont représentés par des bandes à peu près verticales et l'échelle des $\lambda_{1}$ varie dans le même sens que l'échelle des magnitudes absolues.

Les deux composantes de chaque étoile double sont toujours jointes par une droite et le nom de l'étoile double est inscrit à côté de la composante la plus brillante (de même pour les Fig. 4 et 6 ). 
mination du paramètre $\Phi_{\mathrm{b}}$ pour les étoiles $\mathrm{O}$ et B alors que les coordonnées se mesurent facilement pour les naines $\mathrm{A}$ et $\mathrm{F}$ dont beaucoup, plus proches de nous, n'ont pas à subir cette absorption.

Aussi diviserons-nous l'exposé en deux parties : l'étude des étoiles des types A-F et celle des types O-B, en nous bornant dans tous les cas aux classes de luminosité IV et V. Il sera fait usage d'une série de données et de résultats extraits d'un important travail de Jacques Berger, actuellement en cours et concernant la classification des étoiles doubles largement séparées (I), ainsi que de déterminations non encore publiées, faites par Lucienne Divan.

\section{LES ÉTOILES DES TYPES A ET F}

\section{Elles sont représentées par les points d'un volume entourant $\Sigma$.}

Un certain nombre d'étoiles de ces types ont été soigneusement classées : il s'agit d'étoiles simples ou doubles assez proches et situées dans des régions de l'espace dépourvues d'absorption. Leurs points figuratifs sont représentés, Fig. 3b, en projection sur le plan STU (Fig. 2) parallèle à $\lambda_{1} \mathrm{D}(2)$. Nous allons montrer que, dans l'espace, ces points figuratifs se répartissent, au voisinage de $\Sigma$, dans un volume dont l'épaisseur est de beaucoup supérieure à celle qui correspond aux erreurs de détermination des paramètres.

On voit que la Figure 2 que la partie de la surface $\Sigma$ relative aux types spectraux et aux classes de luminosité considérés peut être assimilée à une surface cylindrique dont les génératrices sont les lignes séparant les diverses classes spectrales (ce sont pratiquement des droites parallèles au plan $\lambda_{1} D$ ) : faisons donc une section de $\Sigma$ par un plan xy perpendiculaire à ces génératrices (une partie de la trace xy se voit sur la Fig. $3 \mathrm{~b}$ ) et projetons les points représentatifs sur cette section droite du cylindre (Fig. 4). Ces points apparaissent bien comme disséminés dans un volume limité par deux surfaces à peu près parallèles à $\Sigma$ et situées de part et d'autre (leurs sections par la figure sont les courbes en trait discontinu); ces deux surfaces sont séparées par une distance de l'ordre de 0,30 (mesurée suivant l'axe des $\Phi_{\mathrm{b}}$ ), c'est-à-dire dix fois plus grande que l'erreur moyenne sur une bonne détermination de $\Phi_{b}, 0,03$.

D'autre part, L. Divan a classé I3 étoiles étudiées par N. Roman, 5 strong-lined et 8 weaklined (résultats non encore publiés) : les strong-lined semblent se placer en moyenne plus à droite $\left(\Phi_{\mathrm{b}}\right.$ plus grand) que les weak-lined dans le diagramme de la Fig. 4 , les dernières restant plus voisines de la région de l'espace réservée aux étoiles à grande vitesse. La division des étoiles entre strong-lined et weak-lined serait une manifestation visible sur le spectre des raies, de la complexité des spectres que révèle la spectrophotométrie du fond continu.

Calcul des magnitudes absolues. Ainsi donc trois paramètres sont nécessaires pour classer les étoiles $\mathrm{A}$ et $\mathrm{F}$. Mais on peut se demander si ces trois paramètres suffisent pour caractériser complètement les propriétés générales de ces étoiles. Nous allons voir qu'ils permettent en tous cas de calculer leur magnitude absolue.

La méthode employée par J. Berger pour faire ce calcul est la suivante : les différences de magnitude absolue entre les composantes d'une étoile multiple, étant déterminées par photométrie, sont connues avec une précision beaucoup plus grande que les magnitudes absolues

(I) Les premiers résultats de ce travail ont déjà été mentionnés [2].

Les résultats concernant la classification d'une vingtaine d'étoiles doubles sont contenus dans [3].

(2) La disposition un peu particulière des figures $3 \mathrm{a}, 3 \mathrm{~b}$ est expliquée dans la légende de cette figure. Cette disposition n'a pas d'intérêt particulier actuellement, mais elle en aura pour la seconde partie de l'exposé [5]. 
d'étoiles simples déduites de mesures de parallaxes; de plus, elles ont peu de chances d'être affectées par l'absorption interstellaire.

Aussi commence-t-il par déterminer, à partir des différences de magnitude de quelques étoiles doubles préalablement classées, la loi de variation de la magnitude absolue dans la portion

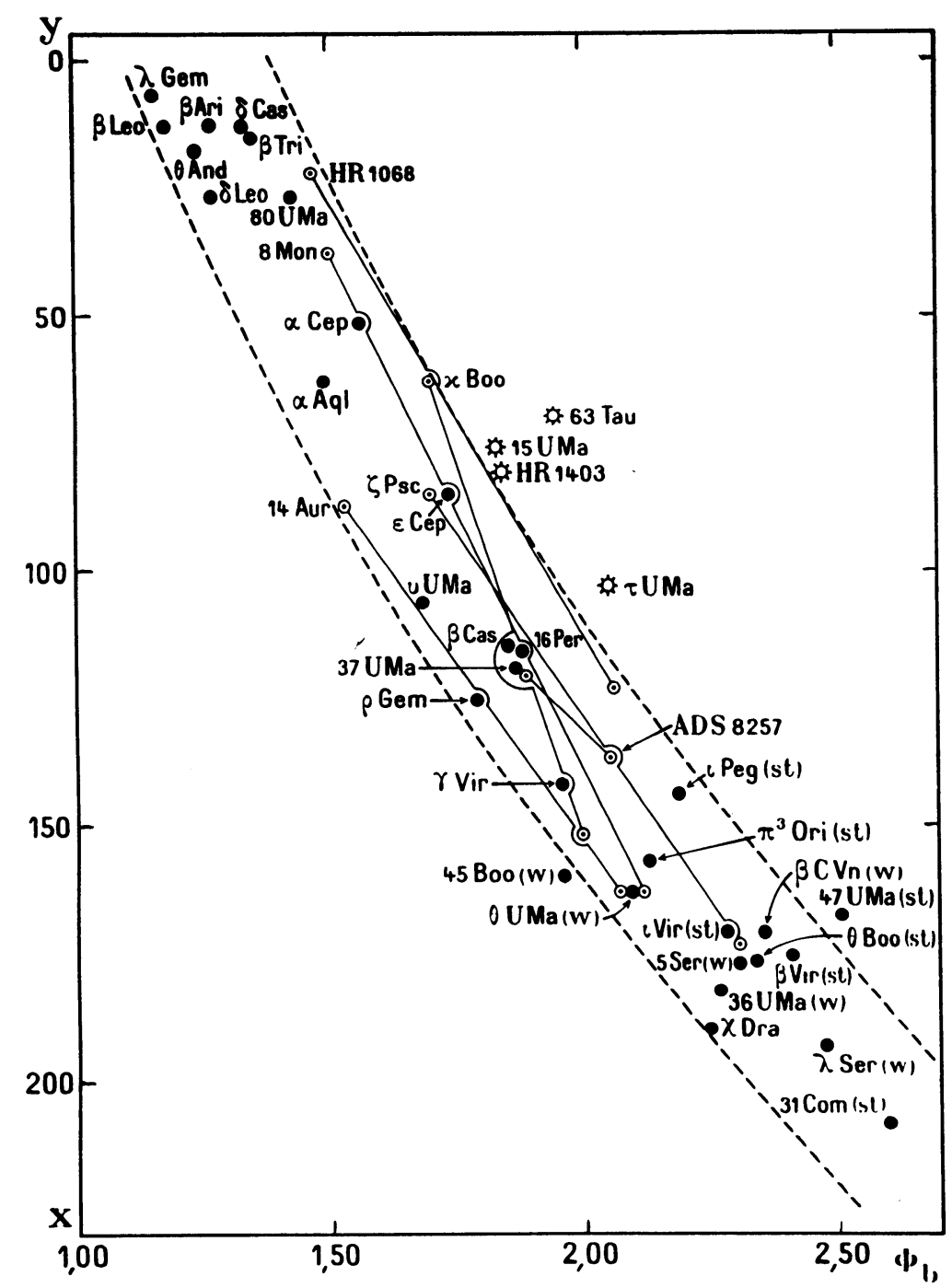

Figure 4. Projection des points figuratifs sur le plan xy (dont la trace est en partie visible sur la Fig. 3 b).

Les points figuratifs représentés se voient en projection sur le plan $\lambda_{1} D$ sur la Fig. 3 b.

Les symboles $w$ et st désignent les étoiles weak-lined et strong-lined de Miss Roman.

Le volume qui contient les divers points figuratifs a une épaisseur très grande si l'on y inclut les étoiles à raies métalliques (*).

considérée de l'espace $\lambda_{1} \mathrm{D} \Phi_{\mathrm{b}}$ (pour simplifier nous appellerons désormais espace $\mathrm{E}$ cet espace $\lambda_{1} \mathrm{D} \Phi_{\mathrm{b}}$ ). La connaissance de quelques magnitudes (déterminées à partir de parallaxes) permettra de fixer ensuite le zéro de l'échelle des magnitudes absolues. 
Soit donc $\Delta \mathrm{M}$ la différence de magnitude absolue entre des étoiles représentées par deux points voisins de l'espace $\mathrm{E}$.

Nous pouvons écrire :

$$
\Delta \mathrm{M}=\frac{\mathrm{o} \mathrm{M}}{\mathrm{a} \lambda_{1}} \Delta \lambda_{1}+\frac{\mathrm{a} \mathrm{M}}{\mathrm{a} \mathrm{D}} \Delta \mathrm{D}+\frac{\mathrm{o} \mathrm{M}}{\mathrm{a} \Phi_{\mathrm{b}}} \Delta \Phi_{\mathrm{b}}
$$

où $\Delta \lambda_{1}, \Delta \mathrm{D}, \Delta \Phi_{\mathrm{b}}$ représentent les différences des coordonnées de ces deux points.

Les six étoiles doubles du tableau I ont été classées avec soin et l'on peut écrire six équations analogues à (I). Dans ce système, les quantités $\Delta \lambda_{1}, \Delta \mathrm{D}, \Delta \Phi_{\mathrm{b}}$ et $\Delta \mathrm{M}$ sont connues (colonnes 2, 3, 4 et 5 du tableau $\mathrm{I}$ ) et l'on peut en déduire les valeurs moyennes des dérivées partielles $\frac{\mathrm{a} M}{\mathrm{a} \lambda_{1}}, \frac{\mathrm{\partial M}}{\mathrm{aD}}, \frac{\mathrm{\partial} \cdot \mathrm{M}}{\mathrm{\partial} \Phi_{\mathrm{b}}}$ dans la région de l'espace $\mathrm{E}$ qui contient les points figuratifs des six couples (Fig. 3 b et Fig. 4).

La résolution par la méthode des moindres carrés de ce système de six équations à trois inconnues conduit aux valeurs suivantes

$$
\frac{\mathrm{a} M}{\mathrm{a} \lambda_{1}}=+0.094 \quad \frac{\mathrm{a} M}{\mathrm{aD}}=-\mathrm{I} 5 . \mathrm{I} 8 \quad \frac{\mathrm{aM}}{\mathrm{a} \Phi_{\mathrm{b}}}=-3.88
$$

\begin{tabular}{|c|c|c|c|c|c|}
\hline Étoile & $\lambda_{1}-3700$ & $\mathrm{D}$ & $\Phi_{\mathrm{b}}$ & $\Delta \mathrm{M}$ observé & $\Delta \mathrm{M}$ calculé \\
\hline 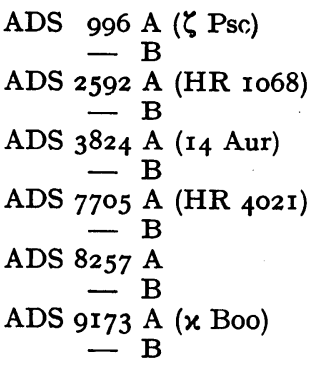 & $\begin{array}{l}67 \\
68 \\
70 \\
72 \\
56 \\
70 \\
64 \\
67 \\
57 \\
64 \\
64 \\
65\end{array}$ & $\begin{array}{l}0.35 \\
0.14 \\
0.47 \\
0.23 \\
0.40 \\
0.16 \\
0.34 \\
0.34 \\
0.27 \\
0.27 \\
0.41 \\
0.20\end{array}$ & $\begin{array}{l}1.70 \\
2.30 \\
1.47 \\
2.06 \\
1.53 \\
2.07 \\
1.97 \\
1.82 \\
2.05 \\
1.88 \\
1.70 \\
1.98\end{array}$ & $\begin{array}{l}\text { I. } 35 \\
2.2\end{array}$ & $\begin{array}{l}\mathrm{I} .54 \\
2.86 \\
0.86 \\
\mathrm{I} .32 \\
2.12\end{array}$ \\
\hline
\end{tabular}

Tableau I

Les valeurs obtenues pour les dérivées partielles permettent de retrouver avec une bonne approximation les valeurs observées pour $\Delta \mathrm{M}$, ainsi que le montre la sixième colonne du tableau I (I).

L'accord obtenu est d'autant plus remarquable que les six couples d'étoiles sont répartis dans un volume relativement vaste de l'espace E.

Pour les deux étoiles doubles 8 Mon et 35 Psc par contre, le calcul et l'observation sont en désaccord : cela peut tenir au fait que, dans les deux cas, la composante brillante est probablement double.

(I) Depuis que ce calcul a été fait une autre étoile double a été classée dont les composantes sont représentées par deux points de la même région de l'espace. La valeur de $\Delta \mathrm{M}$ calculée pour cette étoile à partir des dérivées partielles

\begin{tabular}{|c|c|c|c|c|c|}
\hline Étoile & $\lambda_{1}-3700$ & $\mathrm{D}$ & $\Phi_{\mathrm{b}}$ & $\Delta \mathrm{M}$ observé & $\Delta \mathrm{M}$ calculé \\
\hline $\begin{array}{r}\mathrm{HD} \text { I } 10886 \mathrm{~A} \\
-\quad \mathrm{B}\end{array}$ & $\begin{array}{l}65 \\
69\end{array}$ & $\begin{array}{l}0.35 \\
0.40\end{array}$ & $\begin{array}{l}\mathrm{I} .8 \mathrm{I} \\
\mathrm{I} .5^{\circ}\end{array}$ & 0.8 & 0.82 \\
\hline
\end{tabular}
qui viennent d'être déterminées est en bon accord avec le $\Delta \mathrm{M}$ mesuré : 
Il est facile de vérifier que les valeurs des trois dérivées partielles permettent de calculer la différence de magnitude absolue entre deux étoiles représentées par des points de la région considérée de l'espace $\mathrm{E}$ : la Fig. 5 compare les valeurs calculées pour $\mathrm{M}$, aux valeurs observées pour un certain nombre d'étoiles simples à parallaxe connue, parmi lesquelles figurent des membres des Hyades. Les écarts que met en évidence la Fig. 5 s'expliquent par les erreurs sur les déterminations

I) des coordonnées $\lambda_{1}, D, \Phi_{b}$ (des erreurs moyennes de $2 \AA$ sur $\lambda_{1}, 0.01$ sur $D, 0.05 \operatorname{sur} \Phi_{b}$ amèneront une erreur moyenne de 0.5 sur $\mathrm{M}$, en supposant les dérivées partielles exactement connues);

2) des magnitudes absolues à partir des parallaxes.

On peut donc considérer l'accord entre les valeurs de $\mathrm{M}$ calculées et observées comme très satisfaisant.

Le calcul qui vient d'être fait permet d'évaluer la différence de magnitude absolue entre deux étoiles dont les points représentatifs sont situés aux points d'intersection d'une

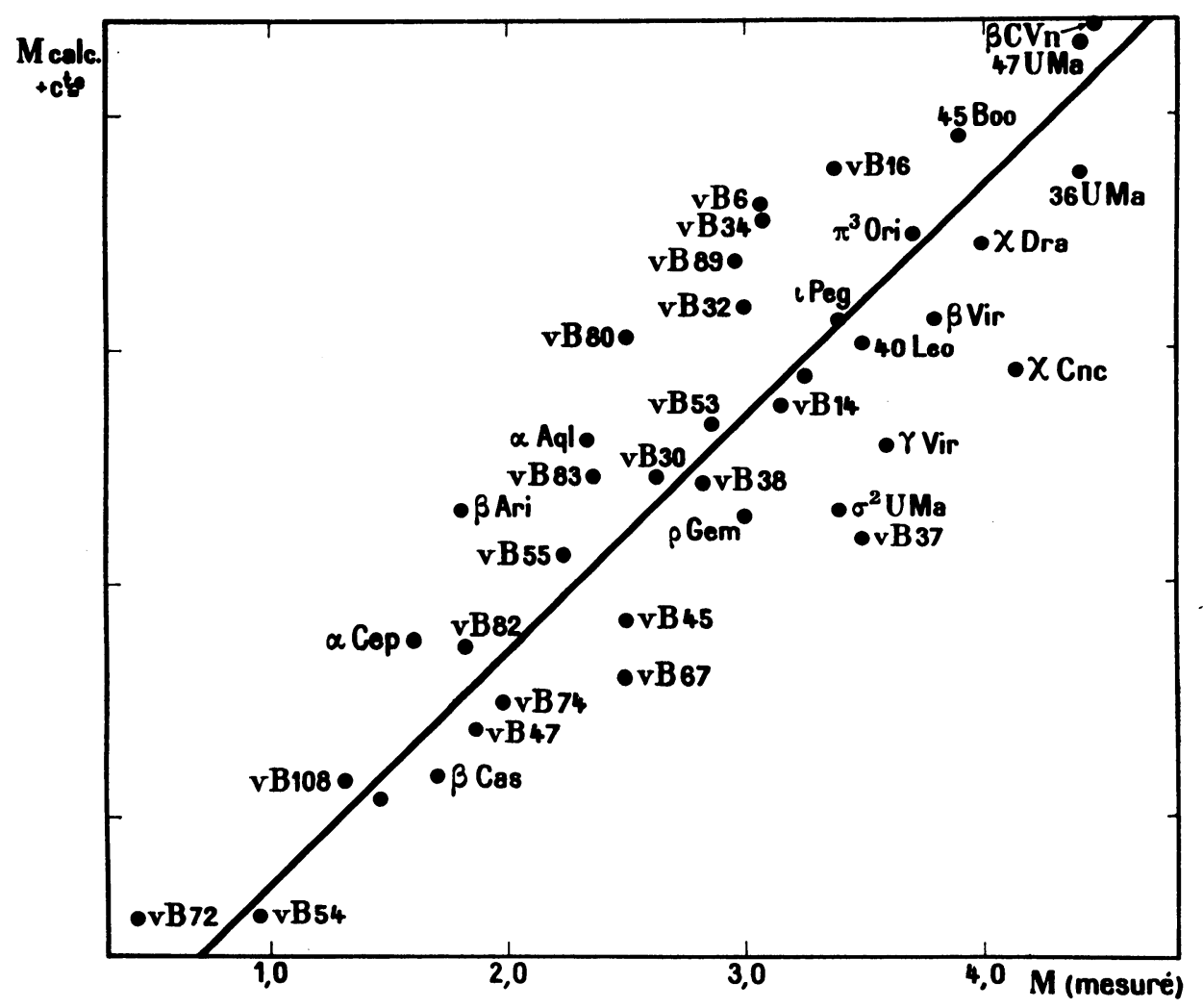

FIgURE 5. Comparaison entre les magnitudes absolues mesurées (abscisses) et les magnitudes absolues calculées (ordonnées).

même parallèle à l'axe $\Phi_{b}$ (mêmes $\lambda_{1}$ et D) avec les deux faces du volume (Fig. 4) : les $\Phi_{b}$ de ces deux points différant de 0.30 environ, leurs magnitudes absolues diffèrent de

$$
0.30 \times \frac{\partial M}{\partial \Phi_{b}}=0.30 \times 3.88=1.2
$$


l'étoile la plus brillante ayant le plus grand $\Phi_{\mathrm{b}}$. La luminosité décroît donc de $\mathrm{I}, 2$ lorsque l'on passe de la face extérieure à la face intérieure (et la décroissance doit se poursuivre si l'on continue dans la même direction puisque l'on arrive dans la région des sous-naines).

En définitive, il apparaît que trois paramètres sont nécessaires pour classer les étoiles des types spectraux considérés et il semble que ces trois parámètres permettront de représenter les propriétés principales des étoiles en question.

LES ÉTOILES DES TYPES O E T B

Etoiles ayant même $\lambda_{1}, D$ et des gradients différents.

On constate souvent des différences notables entre les gradients mesurés $\Phi_{\mathrm{b}}$ de deux étoiles $\mathrm{O}$ ou $B$ ayant sensiblement les mêmes coordonnées $\lambda_{1}$ et $D$. On admettait jusqu'ici que ces écarts pouvaient s'expliquer entièrement par l'absorption interstellaire et que les $\Phi_{\mathrm{b}}$ intrinsèques des deux étoiles étaient les mêmes. Nous allons voir que cette hypothèse n'est pas exacte et que, les $\Phi_{b}$ intrinsèques des deux étoiles peuvent différer notablement, comme dans le cas des types $\mathrm{A}$ et $\mathrm{F}$.

Les étoiles les plus bleues pour chaque type spectral.

Parmi les étoiles d'un type donné (ou ayant une valeur de $\mathrm{D}$ donnée), les plus bleues, c'est-à-dire celles qui ont le plus petit gradient $\Phi_{\mathrm{b}}$, sont celles qui ont le plus de chance de n'avoir pas été affectées par l'absorption interstellaire.

La Fig. 6 montre comment on peut trouver ces étoiles : elle représente la projection sur le plan PSU (Fig. 2) parallèle à $\mathrm{D} \Phi_{\mathrm{b}}$ (et à peu près normal à la portion de la surface $\Sigma$ relative aux étoiles des types $\mathrm{O}$ et $\mathrm{B}$ de luminosité IV et $\mathrm{V}$ ) des points représentatifs d'un certain nombre d'étoiles classées : la partie gauche de la figure $\left(\Phi_{\mathrm{b}}<\mathrm{I}, 00\right)$ correspond aux étoiles des types $\mathrm{O}$ et $\mathrm{B}$, la partie droite $\left(\Phi_{\mathrm{b}}>\mathrm{I}, \mathrm{Oo}\right)$ correspond aux étoiles des types A et $\mathrm{F}$.

On constate que toutes les étoiles $\mathrm{O}$ et $\mathrm{B}$ (et, à fortiori, les étoiles $\mathrm{A}$ et $\mathrm{F}$ ) se placent à droite de la courbe $(C)$ en trait discontinu qui joint les projections des points représentant les étoiles u Ori, $\eta$ Hya, $\beta C$ Mi, $\theta$ Leo. Ceci conduit à admettre que le gradient minimum observable pour chaque valeur de $\mathrm{D}$ est celui que définit la courbe $(\mathrm{C})$ : ces gradients correspondraient à des étoiles exemptes d'absorption interstellaire. Mais nous allons voir que ce ne sont pas les seules et que, parmi les étoiles $\mathrm{O}$ et $\mathrm{B}$ dont le gradient est supérieur à celui que définit (C), certaines n'ont pas davantage subi d'absorption.

Les étoiles doubles. - La Figure 6 représente principalement les projections de points figuratifs d'étoiles doubles classées par J. Berger : les couples de points correspondants sont joints par une droite en trait plein et le nom de l'étoile est marqué auprès de la composante brillante. Si le rayonnement d'un de ces couples a traversé un nuage interstellaire, les deux gradients sont augmentés d'une même quantité : la correction d'absorption (en supposant qu'elle soit connue) se ferait donc en imprimant aux deux points figuratifs de l'étoile double une même translation vers la gauche, parallèlement à l'axe des $\Phi_{\mathrm{b}}$ et cette translation ne devra jamais les amener à gauche de la courbe $(C)$.

Un certain nombre d'étoiles de l'amas NGC 2264 (désignées par la lettre $\mathrm{W}$ suivie du $\mathrm{n}^{0}$ de la liste de Walker [4]) sont représentées sur la Fig. 6 : elles sont reliées par une ligne 
brisée, en trait discontinu. Les étoiles de cet amas étant soumises à une absorption sensiblement uniforme, la correction de cette absorption se fera également par une translation de l'ensemble vers la gauche, parallèlement à $\Phi_{b}$.

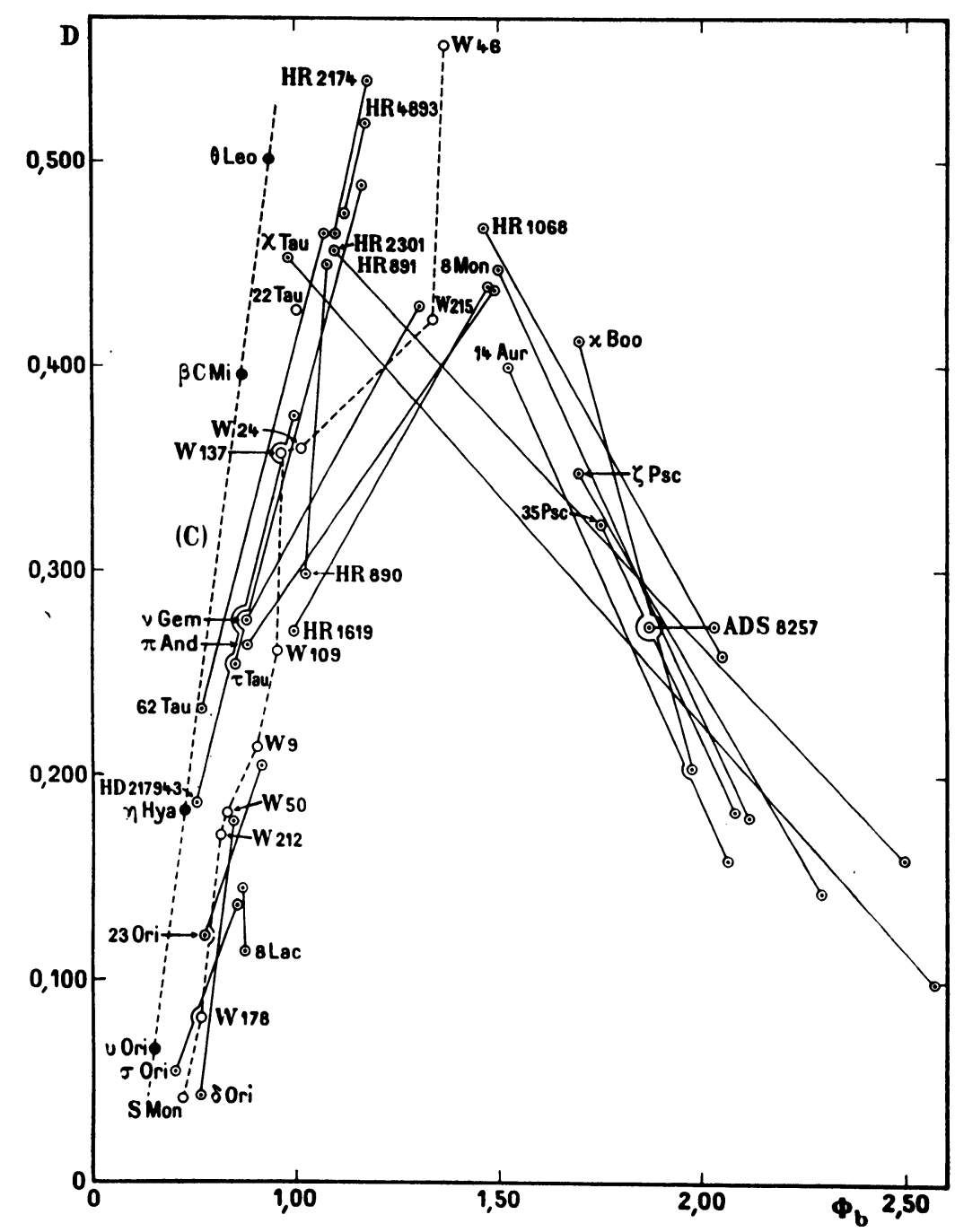

FIGURE 6. Projection des points relatifs à un certain nombre d'étoiles sur le plan PSU parallele aux axes $D$ et $\Phi_{\mathrm{b}}$.

Le plan PSU est à peu près normal à la partie de la surface relative aux étoiles B. La ligne en trait discontinu (C) qui joint les projections des étoiles $v$ Ori, $\eta \mathrm{Hya}, \beta \mathrm{C} \mathrm{Mi}, \theta \mathrm{Leo}$, définit la position des étoiles les plus bleues observables pour les diverses valeurs de $\mathrm{D}$.

Les composantes d'une étoile double sont reliées par un trait plein. Les points figuratifs des deux étoiles doubles HD 2 I 7943 et 62 Tau, fortement rougies, ont été déplacés par translation parallèle à l'axe des $\Phi_{b}$ de façon à les corriger à peu près de l'absorption subie : avant le déplacement, le $\Phi_{\mathrm{b}}$ des composantes brillantes était I.OI pour HD 2 I7943 et $\mathrm{I} .44$ pour 62 Tau.

Il est bien visible sur la Fig. 6 que si les étoiles doubles $\sigma$ Ori, 23 Ori, HD 2I7943, $62 \mathrm{Tau}, \nu$ Gem sont supposées rougies par absorption interstellaire (c'est certain dans le cas de HD 2I7943 et 62 Tau) et si l'on corrige leurs gradients de façon à amener la composante brillante 
de chacun des couples sur la courbe (C) (translation de chacun des quatre couples vers la gauche), la composante faible demeurera nettement à droite de (C). De même, si l'on fait subir à toutes les composantes de l'amas NGC 2264 (jointes par un trait discontinu) une translation de 0,09 vers la gauche de façon à amener S Mon sur (C) (c'est la valeur maximum de la correction d'absorption interstellaire que puisse subir cet amas), les étoiles W 9, W 109, W 137, W 24, W 2I5 resteront à droite de (C) (de même W 46 mais il s'agit d'une $A_{3}$ ).

Nous avons ainsi une série d'exemples d'étoiles non rougies et dont le gradient $\Phi_{b}$ est supérieur au gradient minimum défini par la courbe (C). On voit en particulier très bien (Fig. 6 ) que le compagnon de $\sigma$ Ori a un gradient supérieur à celui de $\eta$ Hya bien qu'étant de type moins avancé; de même, W 24 a un gradient supérieur à celui de $\beta$ CMi bien qu'étant de type moins avancé; W 2I5 a un gradient très nettement plus grand que celui de la composante 22 Tau des Pléiades, ces deux étoiles ayant pratiquement mêmes coordonnées $\lambda_{1}$ et $\mathrm{D}$.

Donc, dans l'espace $E$ les étoiles $B$ ne se placent pas sur la surface mais dans une couche voisine de $\Sigma$. La Figure 6 montre que l'épaisseur de cette couche mesurée parallèlement à l'axe $\Phi_{b}$ ne serait pas inférieure à o, ro pour $\mathrm{D}<0,200$ (types $\mathrm{O}$ à $\mathrm{B}_{4}$ ) mais qu'elle augmente rapidement avec $\mathrm{D}$ (c'est-à-dire lorsqu'on va vers le type $\mathrm{A}$ ).

\section{O N C L U S I O N}

Il semble donc que pour une classification précise des étoiles de tous les types compris entre $\mathrm{O}$ et $\mathrm{F}$ et des classes de luminosité $\mathrm{IV}$ et $\mathrm{V}$, trois paramètres soient nécessaires. Ces trois paramètres définiraient les propriétés principales des étoiles (une démonstration partielle de ce fait a été donnée pour les types $\mathrm{A}$ et $\mathrm{F}$ ).

$\mathrm{La}$ " dispersion cosmique " serait donc la conséquence directe du manque de précision de la classification employée jusqu'ici.

Avec une classification précise, exigeant trois paramètres quantitatifs, une magnitude absolue définie correspondrait à chaque type d'étoile et la " dispersion cosmique " disparaîtrait:

\section{RÉ F E R E N E S}

[I] Chalonge D., A. J. U.R.S.S. 33, 490, 1956.

[2] Chalonge D., Ricerche Astronomiche, 5, 345, 1958.

[3] Berger J., J. des Obs. 41, 105, 1958.

[4] Walker M. F., Ap. J. suppl. 2, 365, 1957.

[5] Berger J., Chalonge D., Divan L., Fringant A.-M., Westerlund B., J. des Obs., 41, 1oo. 1958. 